\title{
Directly Characterizing the Relative Strength and Momentum Dependence of Electron-Phonon Coupling Using Resonant Inelastic X-Ray Scattering
}

\author{
T. P. Devereaux, ${ }^{1,2}$ A. M. Shvaika, ${ }^{3}$ K. Wu, ${ }^{1}$ K. Wohlfeld, ${ }^{4}$ C. J. Jia, ${ }^{1}$ Y. Wang, ${ }^{1}$ B. Moritz, ${ }^{1}$ \\ L. Chaix, ${ }^{1}$ W.-S. Lee, ${ }^{1}$ Z.-X. Shen, ${ }^{1,2,5}$ G. Ghiringhelli, ${ }^{6}$ and L. Braicovich ${ }^{6}$ \\ ${ }^{1}$ Stanford Institute for Materials and Energy Sciences, SLAC National Accelerator Laboratory, \\ 2575 Sand Hill Road, Menlo Park, California 94025, USA \\ ${ }^{2}$ Geballe Laboratory for Advanced Materials, Stanford University, Stanford, California 94305, USA \\ ${ }^{3}$ Institute for Condensed Matter Physics of the National Academy of Sciences of Ukraine, \\ Lviv 79011, Ukraine \\ ${ }^{4}$ Institute of Theoretical Physics, Faculty of Physics, University of Warsaw, \\ Pasteura 5, PL-02093 Warsaw, Poland \\ ${ }^{5}$ Department of Physics and Applied Physics, Stanford University, Stanford, California 94305, USA \\ ${ }^{6}$ CNR-SPIN and Dipartimento di Fisica, Politecnico di Milano, I-20133 Milano, Italy \\ (Received 10 May 2016; revised manuscript received 31 August 2016; published 25 October 2016)
}

\begin{abstract}
The coupling between lattice and charge degrees of freedom in condensed matter materials is ubiquitous and can often result in interesting properties and ordered phases, including conventional superconductivity, charge-density wave order, and metal-insulator transitions. Angle-resolved photoemission spectroscopy and both neutron and nonresonant $\mathrm{x}$-ray scattering serve as effective probes for determining the behavior of appropriate, individual degrees of freedom - the electronic structure and lattice excitation, or phonon dispersion, respectively. However, each provides less direct information about the mutual coupling between the degrees of freedom, usually through self-energy effects, which tend to renormalize and broaden spectral features precisely where the coupling is strong, impacting one's ability to quantitatively characterize the coupling. Here, we demonstrate that resonant inelastic x-ray scattering, or RIXS, can be an effective tool to directly determine the relative strength and momentum dependence of the electron-phonon coupling in condensed matter systems. Using a diagrammatic approach for an eight-band model of copper oxides, we study the contributions from the lowest-order diagrams to the full RIXS intensity for a realistic scattering geometry, accounting for matrix element effects in the scattering cross section, as well as the momentum dependence of the electron-phonon coupling vertex. A detailed examination of these maps offers a unique perspective into the characteristics of electron-phonon coupling, which complements both neutron and nonresonant x-ray scattering, as well as Raman and infrared conductivity.
\end{abstract}

DOI: 10.1103/PhysRevX.6.041019

\section{INTRODUCTION}

The ability to characterize excited states and fundamental excitations in solids remains one of the forefront challenges in condensed matter physics. This challenge is particularly evident in materials that display emergent phases, such as unconventional superconductivity, which stem from intertwined lattice, charge, spin, and orbital degrees of freedom [1]. Typically, simple pictures for excitations derived from well-controlled perturbation expansions do not capture the richness of these materials because of the lack of a small parameter around which to

Published by the American Physical Society under the terms of the Creative Commons Attribution 3.0 License. Further distribution of this work must maintain attribution to the author(s) and the published article's title, journal citation, and DOI.
Subject Areas: Condensed Matter Physics,

Materials Science,

Strongly Correlated Materials expand. This challenge is also evident particularly for excitations around a quantum critical point in correlated materials, where the low-energy degrees of freedom are presumably intertwined. The lack of knowledge of how to truly quantify the strength of couplings for all momenta throughout the Brilloiun zone (BZ) has been one of the major roadblocks to understanding the complex phase diagrams that typically emerge near phase boundaries between various electronic, magnetic, or lattice instabilities.

In the last decade, resonant inelastic x-ray scattering (RIXS) has enabled seminal progress in the understanding of fundamental excitations in correlated materials [2]. By exploiting the role of strong spin-orbit coupling in $2 p$ core levels in $\mathrm{Cu} L$-edge RIXS, it has been shown recently that spin-flip excitations can be probed across a wide variety of materials, complementing RIXS studies of charge transfer, $d-d$ orbital, and bimagnon excitations down to a nowavailable resolution better than $100 \mathrm{meV}$ [3,4]. Perhaps 
more intriguing, from the perspective of a single tool that can provide complete characterization of charge, orbital, spin, and lattice excitations, excitations off of the elastic line of oxygen $K$-edge that occur at multiples of optical phonon energies have become visible in one-dimensional edge-shared $\mathrm{CuO}$ systems [5], while phonon sidebands have emerged off of $d-d$ excitations measured at the $\mathrm{Cu}$ $L$-edge [6]. Progress has been made with a resolution of about $120 \mathrm{meV}$ at the $\mathrm{Cu} L$-edge, already obtained 10 years ago at the Swiss Light Source (SLS) [4,7]. The possibilities were expanded further less than one year ago by the new standard of $35 \mathrm{meV}$ at the $\mathrm{Cu} L$-edge at the European Synchrotron Radiation Facility (ESRF) [8]. Since the phenomena of emergence necessarily involve the intertwined nature of different degrees of freedom in the excitation spectra, knowledge of the changes of excitation spectra is a goal that is well addressed for present and future RIXS experimental configurations.

Specifically, one of the outstanding issues in condensed matter physics is to determine the strength of the coupling of electrons to lattice excitations across the entire BZ. This issue has important ramifications to our understanding of emergent phenomena such as superconductivity and density wave order. For conventional isotropic superconductors, the momentum dependence of the electron-phonon coupling is rather irrelevant to pairing, as all modes contribute to the Cooper instability. However, this is not the case in unconventional (sign-changing) anisotropic superconductors [9-11]. While electron-phonon coupling at large momentum transfers that connects momentum points with different signs of the superconducting gap leads to a suppression of superconductivity, scattering that involves small momentum transfers always enhances superconducting pairing [12]. Likewise, it has become accepted that for dimensions greater than 1, the momentum dependence of the electron-phonon coupling $g(\mathbf{q})$ largely controls the ordering wave vector [13]. Therefore, a determination of $g(\mathbf{q})$ is highly desirable on rather general grounds.

While Raman and optical measurements can extract the coupling $\lambda$ at the zone center [14-16], a reliable method for extracting the strength of lattice couplings for all momenta to date has been inferred indirectly from angle-resolved photoemission (ARPES) experiments and, more directly, from inelastic neutron or high-energy nonresonant $\mathrm{x}$-ray scattering, which couple directly to lattice vibrations [11,17-22]. While ARPES probes electrons at well-defined electron momenta in the BZ, a sum over phonons at all bosonic momenta contributes to renormalization effects detected as "kinks" in dispersion, or abrupt changes of spectral linewidths at the energies of the lattice modes. This method precludes a momentum-resolved way to detect $g(\mathbf{q})$ throughout the BZ.

On the other hand, momentum-dependent coupling can be determined from line-shape fitting of $\mathrm{x}$-ray or neutron scattering. However, these techniques present a fundamental challenge to the extraction of lattice coupling itself. Because of the scattering process that couples to the atomic degrees of freedom, lattice vibrations are particularly sharp and easy to detect when the lattice coupling to electrons is weak. In contrast, when the coupling is large, as seen, for example, in bond-stretching modes in the cuprates or near charge-density wave instabilities, phonon line shapes become broad in both energy and momentum, which interferes with other phonons [20]. Thus, the region of energy and momentum where the lattice coupling is strongest tends to also be the region where a direct extraction of the coupling is most difficult. Therefore, a complementary method that can be used to determine coupling in this region is desirable.

As suggested in earlier studies [5,23-25], RIXS offers a unique insight into the momentum dependence and magnitude of electron-phonon coupling. In this paper, we examine this selectivity of the light-scattering process to determine how and which phonons can be resonantly excited during a resonant $\mathrm{x}$-ray process, and the resulting information that can be obtained about the relative strength and momentum dependence of electron-phonon coupling. We primarily consider an $L$-edge resonant x-ray scattering, where a core electron is excited into the conduction band, and we assume that the most interesting phonons are those that couple to mobile conduction, valence electrons, or holes.

We note that this is different than the situation considered in Ref. [24], which considered the coupling of phonons to a single localized electron at the core-hole site in Mott insulators. The treatment of Holstein phonons coupled to a single electron can be carried out exactly, and it gives rise to multiple phonon Franck-Condon features in RIXS $[6,24]$, in analogy to the line-shape features observable in angle-resolved photoemission for a single hole in a Mott insulator [26]. However, when more than one itinerant electron is considered, the many-body polaron is more difficult to treat because of the large increase of the Hilbert space of coupled electrons and phonons. This idea has been studied for RIXS using cluster exact diagonalization for one-dimensional insulating systems [5,27].

This paper focuses on RIXS for weakly interacting, itinerant electrons to highlight the variety of momentum dependence that can arise because of electron kinetic energy and different phonon dispersion, as well as generalized electron-phonon couplings. We further consider weak electron-phonon coupling where single-phonon processes are dominant, since multiphonon processes are usually suppressed via mobile conduction electron screening. We neglect effects from direct phonon coupling to core levels, which would primarily only affect the intermediate-state (absorption) profile. The RIXS spectra can be well determined in these limits, allowing for an investigation of momentum-dependent couplings and kinetic energies. We particularly highlight the role of polarization and selection rules of the light-electron-phonon coupling, which are already well characterized only at the zone center in Raman measurements. Specifically, we find that RIXS can provide detailed clues in regions of momentum space with strong 
electron-phonon coupling with atomic specificity, to further complement neutron and IXS measurements.

\section{EXPRESSIONS FOR PHONON CONTRIBUTIONS TO RIXS}

Since we are interested in the generic ways in which phonons can be coupled in RIXS, we consider weak electron-phonon coupling and examine the one-phonon contribution to RIXS (Figs. 1 and 2). While other considerations involving the ratio of the main intensities to satellites offer a way to quantify electron-phonon coupling for generic-model Hamiltonians [5,24], our approach follows an extension of resonant Raman scattering from phonons, extended to arbitrary momentum transfers and different x-ray resonant edge processes. We examine the interplay of light polarization configurations with specific momentum-dependent electron-phonon coupling for different classes of coupling (for example, deformation, piezoelectric, and electrostatic), for some oxygen modes common to perovskites. Here, we neglect the role of Coulomb interactions, both among and between the valence electrons and the core hole. While the role of Coulomb interactions is crucial for indirect RIXS processes [2], and for couplings at the BZ center, for larger momentum processes, it plays only a minor role $[11,28,29]$.

The phonon branches are characterized by a frequency $\Omega_{\nu}(\mathbf{q})$ for each mode $\nu$, as well as a generalized coupling to electrons $g_{\nu}^{\alpha, \beta}(\mathbf{k}, \mathbf{q})$. Here, $g_{\nu}^{\alpha, \beta}(\mathbf{k}, \mathbf{q})$ denotes the coupling of phonon mode $\nu$ to an electron in band $\alpha$ carrying momentum $\mathbf{k}$ scattering into band $\beta$ with momentum $\mathbf{k}+\mathbf{q}$. The total RIXS response contains two contributions from these two diagrams, $\chi=\chi_{\text {bare }}+\chi_{\text {phonon, }}$, each of which depends upon $\mathbf{k}_{\mathbf{i}}, \mathbf{k}_{\mathbf{f}}, \mathbf{q}, \omega_{i}, \omega_{f}, \Omega$, where $\mathbf{q}=\mathbf{k}_{\mathbf{i}}-\mathbf{k}_{\mathbf{f}}$ and $\Omega=\omega_{i}-\omega_{f}$.

For the bare loop contribution (Fig. 1), we have [30]

$$
\begin{aligned}
\chi_{\text {bare }}^{\alpha, \beta}\left(\mathbf{k}_{\mathbf{i}}, \mathbf{k}_{\mathbf{f}}, \mathbf{q} \mid \omega_{i}, \omega_{f}, \Omega\right)= & \frac{1}{N} \sum_{\mathbf{p}}\left[\mathbf{e}_{\mathbf{i}} \cdot \mathbf{d}_{\alpha, \beta}\left(\mathbf{p}+\mathbf{q}, \mathbf{p}-\mathbf{k}_{\mathbf{f}}\right)\right]\left[\mathbf{e}_{\mathbf{f}} \cdot \mathbf{d}_{\beta, \alpha}\left(\mathbf{p}-\mathbf{k}_{\mathbf{f}}, \mathbf{p}\right)\right] \\
& \times\left[\mathbf{e}_{\mathbf{i}} \cdot \mathbf{d}_{\alpha, \beta}\left(\mathbf{p}-\mathbf{k}_{\mathbf{f}}, \mathbf{p}+\mathbf{q}\right)\right]\left[\mathbf{e}_{\mathbf{f}} \cdot \mathbf{d}_{\beta, \alpha}\left(\mathbf{p}, \mathbf{p}-\mathbf{k}_{\mathbf{f}}\right)\right] \\
& \times \int_{-\infty}^{+\infty} d \omega[f(-\omega-\Omega)-f(-\omega)]\left|G_{\beta}\left(\mathbf{p}-\mathbf{k}_{\mathbf{f}}, \omega-\omega_{f}\right)\right|^{2} \\
& \times\left[-\frac{1}{\pi} \Im G_{\alpha}(\mathbf{p}, \omega)\right]\left[-\frac{1}{\pi} \Im G_{\alpha}(\mathbf{p}+\mathbf{q}, \omega+\Omega)\right],
\end{aligned}
$$

where $\Im$ denotes the imaginary part and $\alpha, \beta$ denote the conduction and core electron, respectively, appropriate for a direct RIXS transition. The matrix elements generally depend on the incident and scattered photon momentum $\left(\mathbf{k}_{\mathbf{i}, \mathbf{f}}\right)$, energy $\left(\omega_{i, f}\right)$, and polarizations $\mathbf{e}_{\mathbf{i}, \mathbf{f}}$, respectively, including the light dipole couplings $\mathbf{d}_{\alpha, \beta}(\mathbf{k}, \mathbf{p})$ involving

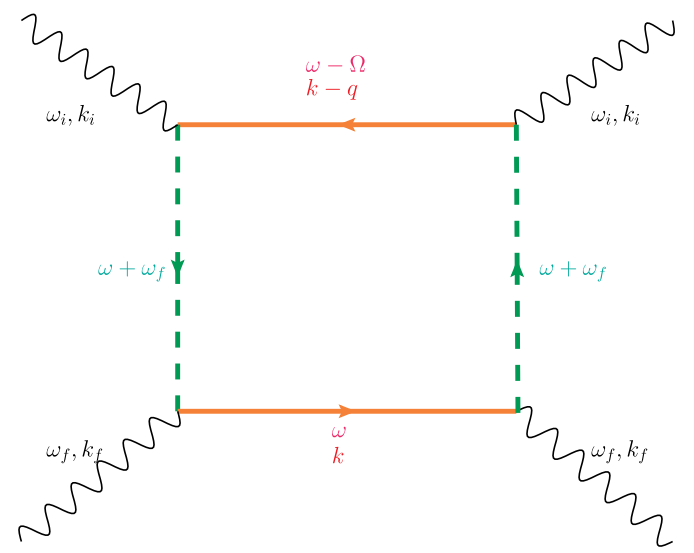

FIG. 1. Electron-hole contribution to RIXS (bare diagram). The dotted lines denote the $\mathrm{Cu} 2 p$ hole, and solid lines denote the $3 d$ conduction electrons. Notation for the vertices has been suppressed. the resonant photoexcitation of a $\beta$ core hole with momentum $\mathbf{p}$ into the $\alpha$ band with momentum $\mathbf{k}$.

As a concrete example, our target will be an understanding of lattice coupling in the cuprates, to which end we consider a $\mathrm{Cu} L$-edge scattering process where a $\mathrm{Cu} 2 p$ core electron is photoexcited into the $3 d$ valence band at a resonant energy around $931 \mathrm{eV}\left(\mathrm{Cu} L_{3}\right.$-edge $)$. However, the formalism will work equally well for other resonant excitations involving core electrons and valence states, such as the oxygen $K$-edge.

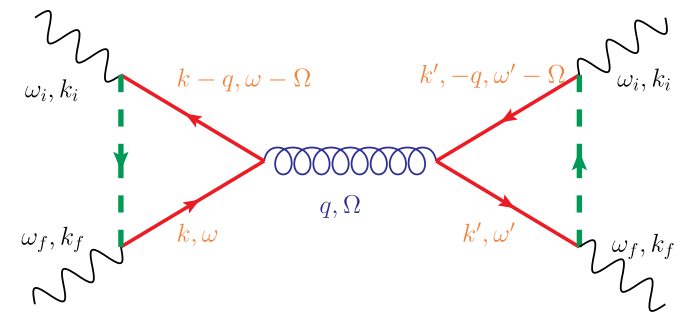

FIG. 2. Leading-order one-phonon contribution to RIXS. The dotted lines denote the $\mathrm{Cu} 2 p$ hole, solid lines denote the $3 d$ conduction electrons, and the circular line denotes the phonon. Notation for the vertices has been suppressed. 
We only consider particle-hole excitations in the $\mathrm{Cu}-\mathrm{O}$ hybridized band that cuts through the Fermi level. This choice allows us to write $G_{3 d}(\mathbf{p}, \omega)=$ $\left|\phi_{3 d}(\mathbf{p})\right|^{2}\left[\omega-\epsilon_{\mathbf{p}}-i \delta_{\mathbf{p}}\right]^{-1}$, with $\epsilon_{\mathbf{p}}$ the eigenenergies, $\phi_{3 d}(\mathbf{p})$ the $\mathrm{Cu} 3 d_{x^{2}-y^{2}}$ projected character of the band, and $\delta_{\mathbf{p}}=\gamma_{e} \operatorname{sign}\left(\epsilon_{\mathbf{p}}\right)$. Expressions for these functions can be determined by Wannier downfolding in densityfunctional-based approaches, or via tight-binding parametrizations for free electrons or exact diagonalization studies for correlated models. In addition, expressions for the momentum-dependent electron-phonon coupling constants $g_{\nu}^{3 d}(\mathbf{p}, \mathbf{q})$ can largely be determined via the same methods. The localized core $\mathrm{Cu} 2 p$ orbitals are represented by $G_{2 p}(\mathbf{p}, \omega)=\left[\omega-E_{2 p, 3 d}+i \Gamma\right]^{-1}$, having an energy $E_{2 p, 3 d}$ measured between the bottom of the $\mathrm{Cu} 3 d$ level and the $\mathrm{Cu}$ $2 p L_{3}$ level, and a phenomenological core-hole lifetime $1 / \Gamma$. One can show that such a resonance approximation for the $2 p$ propagator (which neglects the contributions from other edges) works well for RIXS at either the $\mathrm{Cu} L_{2}$ or $L_{3}$ edge $[31,32]$. As shown in these references and by a number of other recent works, the effect of the core hole does make qualitative changes to the overall resonance profile of RIXS. However, the momentum dependence of the loss profile in RIXS is not altered very much by the presence of a core hole as the effect is largely localized in space. While we do note that a core-hole interaction that extends, for example, from the core copper site to the ligands in transition-metal oxides could have an impact on our considerations, the effect for core-level spectroscopies is expected to be quite small for realistic parameters in transition-metal oxides. Our approximation should be correct as long as the core-level photoexcited electron does not recombine far away from the site in which it is created. The polarization selection rules, as well as the overall momentum dependence, will remain more or less unaffected, and thus the neglect of the core-hole interaction can be justified. Nevertheless, we recognize that further considerations need to made for the case when the photoexcited electron is localized and local multiplet states are affected by the core hole.

The limit $\gamma_{e} \rightarrow 0$ leads to the collapse of the frequency integral. After substitution of the expressions for the Green functions, one obtains

$$
\begin{aligned}
\chi_{\text {bare }}\left(\mathbf{k}_{\mathbf{i}}, \mathbf{k}_{\mathbf{f}}, \mathbf{q} \mid \omega_{i}, \omega_{f}, \Omega\right)= & \frac{1}{N} \sum_{\mathbf{p}}\left[\mathbf{e}_{\mathbf{i}} \cdot \mathbf{d}_{3 d, 2 p}\left(\mathbf{p}+\mathbf{q}, \mathbf{p}-\mathbf{k}_{\mathbf{f}}\right)\right]\left[\mathbf{e}_{\mathbf{f}} \cdot \mathbf{d}_{2 p, 3 d}\left(\mathbf{p}-\mathbf{k}_{\mathbf{f}}, \mathbf{p}\right)\right] \\
& \times\left[\mathbf{e}_{\mathbf{i}} \cdot \mathbf{d}_{2 p, 3 d}\left(\mathbf{p}-\mathbf{k}_{\mathbf{f}}, \mathbf{p}+\mathbf{q}\right)\right]\left[\mathbf{e}_{\mathbf{f}} \cdot \mathbf{d}_{3 d, 2 p}\left(\mathbf{p}, \mathbf{p}-\mathbf{k}_{\mathbf{f}}\right)\right] \\
& \times\left\{f\left[-\epsilon_{\mathbf{p}+\mathbf{q}}\right]-f\left[-\epsilon_{\mathbf{p}}\right]\right\} \frac{\left|\phi_{3 d}(\mathbf{p})\right|^{2}\left|\phi_{3 d}(\mathbf{p}+\mathbf{q})\right|^{2}}{\left|\epsilon_{\mathbf{p}}-\omega_{f}-E_{2 p-3 d}+i \Gamma\right|^{2}} \delta\left[\Omega+\epsilon_{\mathbf{p}}-\epsilon_{\mathbf{p}+\mathbf{q}}\right] .
\end{aligned}
$$

By taking the limit $\gamma_{e} \rightarrow 0$, we neglect the multiparticle interactions within the $3 d$ electrons, focusing our attention on a simple understanding of the role of lattice coupling. For fully interacting electrons, one can still proceed by taking Eq. (1) instead of Eq. (2).

As the main focus of this study, the phonon contribution (represented diagrammatically in Fig. 2) can be cast as [30]

$$
\chi_{\text {phonon }}\left(\mathbf{k}_{\mathbf{i}}, \mathbf{k}_{\mathbf{f}}, \mathbf{q} \mid \omega_{i}, \omega_{f}, \Omega\right)=\left.\frac{1}{2 \pi i} \sum_{\nu, \nu^{\prime}} \Lambda_{\nu}^{(1)}\left(\mathbf{k}_{\mathbf{i}}, \mathbf{k}_{\mathbf{f}}, \mathbf{q} \mid \omega_{i}, \omega_{f}, i \zeta\right) \tilde{D}_{\nu, \nu^{\prime}}(\mathbf{q}, i \zeta) \Lambda_{\nu^{\prime}}^{(2)}\left(\mathbf{k}_{\mathbf{i}}, \mathbf{k}_{\mathbf{f}}, \mathbf{q} \mid \omega_{i}, \omega_{f}, i \zeta\right)\right|_{i \zeta \rightarrow \Omega-i 0^{+}} ^{i \zeta \rightarrow \Omega+i 0^{+}},
$$

with $\nu, \nu^{\prime}$ indices running over phonon modes. Here, we neglect the role of phonon-phonon interactions so that the phonon propagator $\tilde{D}_{\nu, \nu^{\prime}}$ can be taken to be diagonal in phonon indices and can be given simply by $2 \omega_{\nu, \mathbf{q}} /$ $\left[(i \zeta)^{2}-\omega_{\nu, \mathbf{q}}^{2}\right]$ in the limit of weak coupling of electrons to phonon modes having frequencies $\omega_{\nu, \mathbf{q}}$. In accordance with Fermi's golden rule, this contribution involves the product of two matrix elements $\Lambda$ and the phonon density of states. In the limit of large core-hole energy $E_{2 p, 3 d}$, the matrix elements can be written as

$$
\begin{aligned}
\Lambda_{\nu}^{(1)}\left(\mathbf{k}_{\mathbf{i}}, \mathbf{k}_{\mathbf{f}}, \mathbf{q} \mid \omega_{i}, \omega_{f}, i \zeta\right)= & \frac{1}{N} \sum_{\mathbf{p}}\left[\mathbf{e}_{\mathbf{i}} \cdot \mathbf{d}_{3 d, 2 p}\left(\mathbf{p}+\mathbf{q}, \mathbf{p}-\mathbf{k}_{\mathbf{f}}\right)\right]\left[\mathbf{e}_{\mathbf{f}} \cdot \mathbf{d}_{2 p, 3 d}\left(\mathbf{p}-\mathbf{k}_{\mathbf{f}}, \mathbf{p}\right)\right] \\
& \times g_{\nu}^{3 d, 3 d}(\mathbf{p}, \mathbf{p}+\mathbf{q}) \int_{-\infty}^{+\infty} d \omega f(-\omega)\left\{-\frac{1}{\pi} \Im G_{3 d}(\mathbf{p}+\mathbf{q}, \omega) \cdot G_{2 p}^{*}\left(\mathbf{p}-\mathbf{k}_{\mathbf{f}}, \omega-\omega_{i}\right) G_{3 d}(\mathbf{p}, \omega-i \zeta)\right. \\
& \left.-\frac{1}{\pi} \Im G_{3 d}(\mathbf{p}, \omega) \cdot G_{2 p}^{*}\left(\mathbf{p}-\mathbf{k}_{\mathbf{f}}, \omega-\omega_{f}\right) G_{3 d}(\mathbf{p}+\mathbf{q}, \omega+i \zeta)\right\}
\end{aligned}
$$

and 


$$
\begin{aligned}
\Lambda_{\nu}^{(2)}\left(\mathbf{k}_{\mathbf{i}}, \mathbf{k}_{\mathbf{f}}, \mathbf{q} \mid \omega_{i}, \omega_{f}, i \zeta\right)= & \frac{1}{N} \sum_{\mathbf{p}^{\prime}}\left[\mathbf{e}_{\mathbf{i}} \cdot \mathbf{d}_{2 p, 3 d}\left(\mathbf{p}^{\prime}-\mathbf{k}_{\mathbf{f}}, \mathbf{p}^{\prime}+\mathbf{q}\right)\right]\left[\mathbf{e}_{\mathbf{f}} \cdot \mathbf{d}_{3 d, 2 p}\left(\mathbf{p}^{\prime}, \mathbf{p}^{\prime}-\mathbf{k}_{\mathbf{f}}\right)\right] \\
& \times g_{\nu}^{3 d, 3 d}\left(\mathbf{p}^{\prime}+\mathbf{q}, \mathbf{p}^{\prime}\right) \int_{-\infty}^{+\infty} d \omega^{\prime} f\left(-\omega^{\prime}\right)\left\{-\frac{1}{\pi} \Im G_{3 d}\left(\mathbf{p}^{\prime}+\mathbf{q}, \omega^{\prime}\right) \cdot G_{2 p}\left(\mathbf{p}^{\prime}-\mathbf{k}_{\mathbf{f}}, \omega^{\prime}-\omega_{i}\right) G_{3 d}\left(\mathbf{p}^{\prime}, \omega^{\prime}-i \zeta\right)\right. \\
& \left.-\frac{1}{\pi} \Im G_{3 d}\left(\mathbf{p}^{\prime}, \omega^{\prime}\right) \cdot G_{2 p}\left(\mathbf{p}^{\prime}-\mathbf{k}_{\mathbf{f}}, \omega^{\prime}-\omega_{f}\right) G_{3 d}\left(\mathbf{p}^{\prime}+\mathbf{q}, \omega^{\prime}+i \zeta\right)\right\}
\end{aligned}
$$

Using the previous Green function definitions, these equations become

$$
\begin{aligned}
\Lambda_{\nu}^{(1)}\left(\mathbf{k}_{\mathbf{i}}, \mathbf{k}_{\mathbf{f}}, \mathbf{q} \mid \omega_{i}, \omega_{f}, i \zeta\right)= & \frac{1}{N} \sum_{\mathbf{p}}\left[\mathbf{e}_{\mathbf{i}} \cdot \mathbf{d}_{3 d, 2 p}\left(\mathbf{p}+\mathbf{q}, \mathbf{p}-\mathbf{k}_{\mathbf{f}}\right)\right]\left[\mathbf{e}_{\mathbf{f}} \cdot \mathbf{d}_{2 p, 3 d}\left(\mathbf{p}-\mathbf{k}_{\mathbf{f}}, \mathbf{p}\right)\right] \\
& \times g_{\nu}^{3 d, 3 d}(\mathbf{p}, \mathbf{p}+\mathbf{q}) \frac{\left|\phi_{3 d}(\mathbf{p})\right|^{2}\left|\phi_{3 d}(\mathbf{p}+\mathbf{q})\right|^{2}}{\epsilon_{\mathbf{p}}-\epsilon_{\mathbf{p}+\mathbf{q}}+i \zeta+i \gamma_{e}} \\
& \times\left[\frac{f\left(-\epsilon_{\mathbf{p}}\right)}{\epsilon_{\mathbf{p}}-\omega_{f}-E_{2 p, 3 d}-i \Gamma}-\frac{f\left(-\epsilon_{\mathbf{p}+\mathbf{q}}\right)}{\epsilon_{\mathbf{p}+\mathbf{q}}-\omega_{i}-E_{2 p, 3 d}-i \Gamma}\right]
\end{aligned}
$$

and

$$
\begin{aligned}
\Lambda_{\nu}^{(2)}\left(\mathbf{k}_{\mathbf{i}}, \mathbf{k}_{\mathbf{f}}, \mathbf{q} \mid \omega_{i}, \omega_{f}, i \zeta\right)= & \frac{1}{N} \sum_{\mathbf{p}^{\prime}}\left[\mathbf{e}_{\mathbf{i}} \cdot \mathbf{d}_{2 p, 3 d}\left(\mathbf{p}^{\prime}-\mathbf{k}_{\mathbf{f}}, \mathbf{p}^{\prime}+\mathbf{q}\right)\right]\left[\mathbf{e}_{\mathbf{f}} \cdot \mathbf{d}_{3 d, 2 p}\left(\mathbf{p}^{\prime}, \mathbf{p}^{\prime}-\mathbf{k}_{\mathbf{f}}\right)\right] \\
& \times g_{\nu}^{3 d, 3 d}\left(\mathbf{p}^{\prime}+\mathbf{q}, \mathbf{p}^{\prime}\right) \frac{\left|\phi_{3 d}\left(\mathbf{p}^{\prime}\right)\right|^{2}\left|\phi_{3 d}\left(\mathbf{p}^{\prime}+\mathbf{q}\right)\right|^{2}}{\epsilon_{\mathbf{p}^{\prime}}-\epsilon_{\mathbf{p}^{\prime}+\mathbf{q}}+i \zeta+i \gamma_{e}} \\
& \times\left[\frac{f\left(-\epsilon_{\mathbf{p}^{\prime}}\right)}{\epsilon_{\mathbf{p}^{\prime}}-\omega_{f}-E_{2 p, 3 d}+i \Gamma}-\frac{f\left(-\epsilon_{\mathbf{p}^{\prime}+\mathbf{q}}\right)}{\epsilon_{\mathbf{p}^{\prime}+\mathbf{q}}-\omega_{i}-E_{2 p, 3 d}+i \Gamma}\right] .
\end{aligned}
$$

Here, we do not assume free electrons, and we keep $\gamma_{e}$ finite.

These matrix elements carry the resonant enhancement of phonon scattering via coupling to electrons. Importantly, they show that the resonance process itself imparts a projection of the electron-phonon coupling to the portion of the coupling involving the photoexcited valence electron, which, in this case, is copper. If we instead focus on the oxygen $K$-edge, the matrix elements would involve the projection onto the oxygen contribution to the hybridized valence band. This is one important facet that already distinguishes RIXS from other measurements and allows RIXS from phonons to have an element selectivity not only for the particle-hole excitations but also for the momentum-dependent phonon coupling to them.

Finally, for the phonon contribution, one obtains

$$
\begin{aligned}
\chi_{\text {phonon }}\left(\mathbf{k}_{\mathbf{i}}, \mathbf{k}_{\mathbf{f}}, \mathbf{q} \mid \omega_{i}, \omega_{f}, \Omega\right)= & \sum_{\nu} \frac{1}{N} \sum_{\mathbf{p}} \frac{1}{N} \sum_{\mathbf{p}^{\prime}}\left[\mathbf{e}_{\mathbf{i}} \cdot \mathbf{d}_{3 d, 2 p}\left(\mathbf{p}+\mathbf{q}, \mathbf{p}-\mathbf{k}_{\mathbf{f}}\right)\right]\left[\mathbf{e}_{\mathbf{f}} \cdot \mathbf{d}_{2 p, 3 d}\left(\mathbf{p}-\mathbf{k}_{\mathbf{f}}, \mathbf{p}\right)\right] g_{\nu}^{3 d, 3 d}(\mathbf{p}, \mathbf{p}+\mathbf{q}) \\
& \times\left|\phi_{3 d}(\mathbf{p})\right|^{2}\left|\phi_{3 d}(\mathbf{p}+\mathbf{q})\right|^{2}\left[\frac{f\left(-\epsilon_{\mathbf{p}}\right)}{\epsilon_{\mathbf{p}}-\omega_{f}-E_{2 p, 3 d}-i \Gamma}-\frac{f\left(-\epsilon_{\mathbf{p}+\mathbf{q}}\right)}{\left.\epsilon_{\mathbf{p}+\mathbf{q}}-\omega_{i}-E_{2 p, 3 d}-i \Gamma\right]}\right. \\
& \times\left[\mathbf{e}_{\mathbf{i}} \cdot \mathbf{d}_{2 p, 3 d}\left(\mathbf{p}^{\prime}-\mathbf{k}_{\mathbf{f}}, \mathbf{p}^{\prime}+\mathbf{q}\right)\right]\left[\mathbf{e}_{\mathbf{f}} \cdot \mathbf{d}_{3 d, 2 p}\left(\mathbf{p}^{\prime}, \mathbf{p}^{\prime}-\mathbf{k}_{\mathbf{f}}\right)\right] g_{\nu}^{3 d, 3 d}\left(\mathbf{p}^{\prime}+\mathbf{q}, \mathbf{p}^{\prime}\right) \\
& \times\left|\phi_{3 d}\left(\mathbf{p}^{\prime}\right)\right|^{2}\left|\phi_{3 d}\left(\mathbf{p}^{\prime}+\mathbf{q}\right)\right|^{2}\left[\frac{f\left(-\epsilon_{\mathbf{p}^{\prime}}\right)}{\epsilon_{\mathbf{p}^{\prime}}-\omega_{f}-E_{2 p, 3 d}+i \Gamma}-\frac{f\left(-\epsilon_{\mathbf{p}^{\prime}+\mathbf{q}}\right)}{\epsilon_{\mathbf{p}^{\prime}+\mathbf{q}}-\omega_{i}-E_{2 p, 3 d}+i \Gamma}\right] \\
& \times \frac{1}{\pi} \Im\left\{\frac{1}{\epsilon_{\mathbf{p}}-\epsilon_{\mathbf{p}+\mathbf{q}}+\Omega+i \gamma_{e}} \tilde{D}_{\nu}(\mathbf{q}, \Omega) \frac{1}{\epsilon_{\mathbf{p}^{\prime}}-\epsilon_{\mathbf{p}^{\prime}+\mathbf{q}}+\Omega+i \gamma_{e}}\right\} .
\end{aligned}
$$


We see that there can be several sources of enhancement from the resonant process. First, if both the $\mathbf{p}$ and $\mathbf{p}+\mathbf{q}$ states are occupied, no scattering can occur since a core electron is Pauli blocked. Therefore, at least one or both of those states must be unoccupied. However, the largest contribution results when only one of the momentum states is unoccupied, so an occupied electron state can refill the core hole.

Since we assume that the phonons are eigenstates indexed by $\nu$, the loss peaks are determined by the phonon mode energies and their dispersion with momentum, multiplied by overall factors that represent the resonant excitation process. This demonstrates how the electronhole pairs created by the process couple to those phonons in a symmetry-dependent way that is sampled by the incident and scattered polarization orientations. This process is analogous to the way in which photon polarization selection rules can be used to probe unconventional superconductivity $[15,28]$.

\section{GENERAL BEHAVIOR OF RIXS FROM PHONONS}

We can examine a few insights from the momentumdependent electron-phonon coupling matrix element. From Eqs. (6)-(8), it is clear that the overall intensity of the phonon contribution to RIXS is governed by the strength and momentum dependence of the electron-phonon coupling $g_{\nu}^{3 d, 3 d}(\mathbf{q})$. We first consider general couplings that determine its momentum dependence.

For electron-phonon coupling of the deformation type, which involves lattice motion that modifies the kinetic energy of electrons via orbital overlaps on adjacent ions, the electron-phonon coupling depends strongly on transferred momentum $\mathbf{q}$, which is largest for large momentum transfers and vanishing in the limit of $\mathbf{q} \rightarrow 0$ [33]. These phonons, such as the longitudinal acoustic modes or the bond-stretching modes in the cuprates, fail to appear in Raman or optical conductivity and likewise would not contribute to RIXS for small momentum transfers via Eqs. (6) and (7). Moreover, the largest RIXS intensity from those phonons will occur for large momentum at the BZ boundaries, where deformation electron-phonon coupling is largest.

On the other hand, for couplings that are driven by modulations of the electrostatic energies via atomic motion, the momentum dependence is biased towards small momentum transfers. These include out-of-plane $c$-axis oxygen vibrations in transition-metal oxides, which show up in Raman and optical measurements and likewise in RIXS at small wave-vector transfers [11].

In addition, any phonons that do not transform according the full irreducible point-group symmetry of the lattice will likewise vanish at the zone center provided that the dipole matrix elements do not depend upon the magnitude of the momenta involved in scattering. Since the dipole matrix element involves photoexcitations of core levels and is thus quite local, this would be a very good approximation. As a consequence, scattering would be suppressed from zonecenter phonons having $B_{1,2}$ or $E_{g}$ symmetries in $D^{4 h}$ systems. This is quite different from resonant optical Raman scattering, where electrons can be nonlocally excited out of the valence band into fully itinerant bands and thus depend on the momenta involved in the scattering process.

We can show that this is the case for $\mathrm{Cu} L$-edge RIXS via the following consideration. Following Ref. [2], the RIXS vertex in the dipole approximation (here, for the process from the initial to intermediate state of RIXS) is

$$
\hat{V}=\frac{1}{\sqrt{N}} \sum_{j=1}^{N} e^{i \mathbf{k}_{\mathrm{i}} \cdot \mathbf{j}} \hat{\mathbf{r}}_{\mathbf{j}} \cdot \mathbf{e}_{\mathbf{i}},
$$

where $\mathbf{k}_{\mathrm{i}}\left(\mathbf{e}_{\mathrm{i}}\right)$ is the incoming photon momentum (polarization), $\mathbf{j}$ is the lattice site, and $\hat{\mathbf{r}}_{\mathbf{j}}$ is the position operator of the electron at site $\mathbf{j}$.

This can be written in the second quantization form (for the transition-metal ion $L$ edge) as

$\hat{V}=\sum_{\mathbf{q}, \alpha, \beta}\left(\mathbf{d}_{2 p \alpha, 3 d \beta} c_{\mathbf{q}+\mathbf{k}_{\mathbf{i}}, 2 p \alpha}^{\dagger} c_{\mathbf{q}, 3 d \beta}+\mathbf{d}_{2 p \alpha, 3 d \beta} c_{\mathbf{q}+\mathbf{k}_{\mathrm{i}}, 3 d \beta}^{\dagger} c_{\mathbf{q}, 2 p \alpha}\right) \mathbf{e}_{\mathrm{i}}$,

where $\alpha(\beta)$ are the quantum numbers of the electron in the $2 p(3 d)$ orbital.

Here, the RIXS dipole operator matrix element $\mathbf{d}_{2 p \alpha, 3 d \beta}$ does not depend on the momentum of the valence or core electrons since it follows from the expression

$$
\hat{\mathbf{r}}_{\mathbf{j}}=\sum_{n, n^{\prime}, \mathbf{l}, \mathbf{m}} c_{\mathbf{j} n}^{\dagger}\left|\phi_{\mathbf{l} n}\right\rangle\left\langle\phi_{\mathbf{l} n}\left|\hat{\mathbf{r}}_{\mathbf{j}}\right| \phi_{\mathbf{m} n^{\prime}}\right\rangle\left\langle\phi_{\mathbf{m} n^{\prime}}\right| c_{\mathbf{j} n}
$$

(where $n$ and $n^{\prime}$ are quantum numbers of the electrons in the $3 d$ or $2 p$ orbitals; $\mathbf{1}, \mathbf{m}$ are lattice sites; and $\left|\phi_{\mathbf{j}, n}\right\rangle$ are Wannier states centered on atom $\mathbf{j}$ ) and the fact that

$$
\left\langle\phi_{\mathbf{1} 2 p \alpha}\left|\hat{\mathbf{r}}_{\mathbf{j}}\right| \phi_{\mathbf{m} 3 d}\right\rangle \simeq \delta_{\mathbf{m} \mathbf{j}} \delta_{\mathbf{l j}} \times \mathbf{f}(\alpha, \beta) \equiv \mathbf{d}_{2 p \alpha, 3 d \beta} .
$$

This result is due to the localized nature of the core hole. This property distinguishes RIXS from optical Raman scattering.

In this paper, where we are just interested in RIXS in the energy range of single phonons, we keep only the $\beta=$ $d_{x^{2}-y^{2}}$ orbital and neglect the other $\mathrm{Cu} 3 d$ orbitals that would appear if we consider larger energy transfers at the $d-d$ excitation lines. Moreover, we are solely interested in the RIXS transitions for which the spin of the $3 d$ electrons is conserved: This is possible when choosing a particular combination of the polarization vectors $\mathbf{e}_{\mathbf{i}}$ and $\mathbf{e}_{\mathbf{f}}$ [31, 34-37]. Thus, assuming that the latter indeed takes place, 
we can neglect the spin quantum number carried by the $3 d$ electron, and we are left with the dipole matrix elements that depend solely on the $2 p$ orbital quantum number: $\mathbf{d}_{2 p \alpha, 3 d \beta} \equiv \mathbf{d}_{2 p \alpha, 3 d}$, where $\alpha=x, y$. We note that in RIXS experiments for cuprates, different momentum transfers are usually realized by changing the relative angle between the incoming photons and the sample. Thus, the incoming and outgoing photon polarizations $\mathbf{e}_{\mathbf{i}, \mathbf{f}}$ may effectively depend on the momentum transfer [36]. We do not consider this effect in the current manuscript.

\section{AN EXAMPLE: EIGHT-BAND Cu-O MODEL}

We can evaluate RIXS for a simple model as given in Refs. [11,22], which derived momentum-dependent electron-phonon couplings for $\mathrm{Cu}-\mathrm{O}$ planar longitudinal acoustic, bond-stretching, and bond-bending modes, as well as $c$-axis apical modes. Besides accounting for the phonon eigenmodes, the model consists of two $\mathrm{Cu}\left(3 d_{x^{2}-y^{2}}\right.$ and $\left.4 s\right)$ orbitals octahedrally coordinated and hybridized with $\mathrm{O}$ $2 p_{x, y}$ orbitals that are $\sigma$ bonded to the $\mathrm{Cu} 3 d_{x^{2}-y^{2}}$ orbital, plus the apical $\mathrm{O} 2 p_{z}$ orbital hybridized with $\mathrm{Cu} 4 s$. Oxygen hopping is taken into account. We finally add the three $\mathrm{Cu} 2 p$ core levels from which electrons are resonantly excited.

For the phonon excitations, we specifically focus on the oxygen modes common to all perovskite oxides. These phonons typically couple to $\mathrm{CuO}$ electrons either electrostatically in the case of $c$-axis phonon modes or via bond deformation for in-plane stretching modes. The forms for the electron-phonon coupling depend strongly on which process is dominant and can further depend strongly on either fermion momentum $\mathbf{k}$ or phonon momentum transfer q. In Ref. [11], these modes were examined, and expressions for their couplings were derived from general symmetry grounds. Their forms can be expressed as

$$
\begin{aligned}
g_{\mathrm{LA}, \mathrm{br}}(\mathbf{k}, \mathbf{q})= & g_{\mathrm{LA}, \mathrm{br}}^{0} \sqrt{\left[\sin ^{2}\left(q_{x} / 2\right)+\sin ^{2}\left(q_{y} / 2\right)\right]} / 2, \\
g_{A 1, B 1}(\mathbf{k}, \mathbf{q})= & g_{A 1, B 1}^{0}\left[\sin \left(k_{x} / 2\right) \sin \left(p_{x} / 2\right) \cos \left(q_{y} / 2\right)\right. \\
& \left. \pm \sin \left(k_{y} / 2\right) \sin \left(p_{y} / 2\right) \cos \left(q_{x} / 2\right)\right] / 2 \\
g_{\text {apex }}(\mathbf{k}, \mathbf{q})= & g_{\text {apex }}^{0}\left[\cos \left(k_{x} / 2\right)-\cos \left(k_{y} / 2\right)\right] \\
& \times\left[\cos \left(p_{x} / 2\right)-\cos \left(p_{y} / 2\right)\right] / 4,
\end{aligned}
$$

with $\mathbf{p}=\mathbf{k}+\mathbf{q}$ and the lattice constant $a$ set to 1 . Here, LA denotes the longitudinal acoustic modes; br the $\mathrm{Cu}-\mathrm{O}$ inplane bond-stretching modes; $A 1, B 1$ the $c$-axis oxygen modes involving charge transfer between $\mathrm{Cu}-\mathrm{O}$ and $\mathrm{O}-\mathrm{O}$, respectively; and apex the apical oxygen modes. The prefactors $g_{\nu}^{0}$ for mode $\nu$ are rather weakly dependent on momenta and are generally set by the orbital content of the bands that are coupled to the lattice modes. Here, we limit our focus on these prefactors to solely setting an overall magnitude relative to each other. For more details, the reader is directed to Ref. [11]. Finally, we take the apical and $A 1, B 1$ modes to be dispersionless phonons of frequencies $\Omega=85,40$, and $35 \mathrm{meV}$, respectively, and the dispersions of the acoustic and breathing modes in $\mathrm{eV}$ as $\Omega_{\mathrm{LA}}=0.015 \sqrt{\left[\sin ^{2}\left(q_{x} / 2\right)+\sin ^{2}\left(q_{y} / 2\right)\right] / 2}$ and $\Omega_{\mathrm{br}}=$ $0.085\left[1-0.18 \sqrt{\sin ^{2}\left(q_{x} / 2\right)+\sin ^{2}\left(q_{y} / 2\right)}\right]$ to match the dispersions in Bi-based bilayer cuprates [21].

We neglect any momentum or angle dependence of the polarization vectors and take them to be constants such that Eqs. (1) and (8) can be simply integrated numerically. Here, we have taken a simple downfolded three-band $\mathrm{Cu}-\mathrm{O}$ tight-binding model with $\mathrm{Cu}-\mathrm{O}$ hopping $t_{p d}=$ $1.6 \mathrm{eV}$ and charge transfer $0.9 \mathrm{eV}$ to mimic the antibonding band structure $\epsilon_{\mathbf{k}}$ of the cuprates, doped for an overall filling $\langle n\rangle \sim 0.84$ [14,16]. Additionally, we have set $\omega_{i}+E_{2 p, 3 d}=0$ since the resonance is quite broad because of the short core-hole lifetime $(\Gamma=0.5 \mathrm{eV})$. The width of all the phonons is set to $5 \mathrm{meV}$, and $\gamma_{e}=0.010 \mathrm{eV}+\Omega$ is taken as the width of the $\delta$ function in Eqs. (1) and (8) to phenomenologically mimic inelastic relaxation in the particle-hole continuum. The resulting RIXS response is shown in Figs. (3-9) for momentum cuts along the BZ $x$ axis. Since the phonons enter additively, we plot them individually, as their intensities can be set from considerations given in the references cited above.

Figure 3 shows the bare contribution from particle-hole excitations to RIXS from Eq. (1). The particle-hole continuum is resonantly enhanced, with the leading edge rapidly dispersing away from the BZ center with Fermi velocity. The overall continuum expands with increasing energy loss and momentum transfer because of the kinematic conditions of particle-hole creation, with the overall strength and sharpness of the continuum being set by the inelastic relaxation $\gamma_{e}$. We note that the continuum dips down close to zero energy transfer around a momentum $(q, 0) \sim 0.4 \pi$, reflecting the weak nesting condition of the band structure and Fermi surface for this momentum transfer.

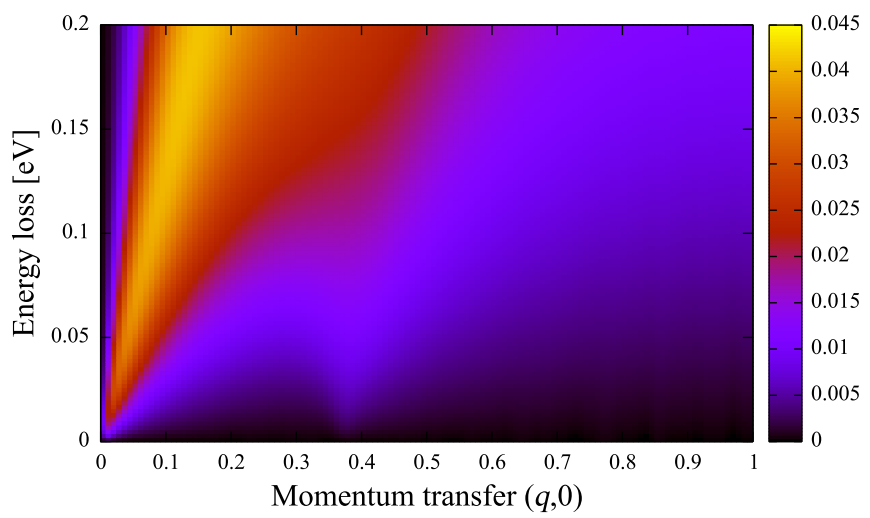

FIG. 3. Bare contribution to RIXS from particle-hole scattering. Momentum transfer is given in units of $\pi / a$. 


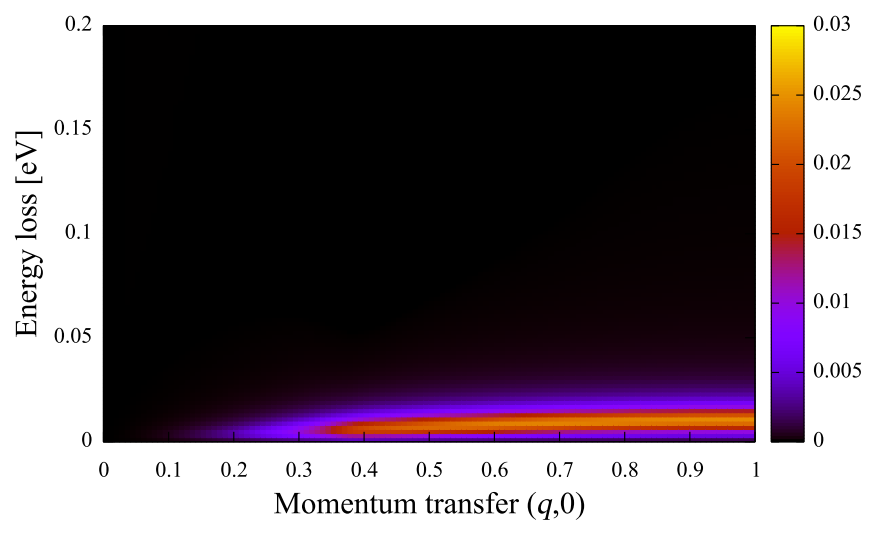

FIG. 4. Longitudinal acoustic phonon contribution to RIXS. Momentum transfer is given in units of $\pi / a$.

Figures 4-8 show the single-phonon contribution to RIXS for the individual phonons listed above. For the acoustic phonon branch (Fig. 4), it is clear that the dispersion for RIXS follows the phonon dispersion, having an intensity that follows the momentum dependence of the electron-phonon coupling $\sim \sin ^{2}(q a / 2)$. The relative strength of the phonon contribution to RIXS compared to the bare response (Fig. 4) is set both by the overall electronphonon coupling and the particle-hole relaxation $\gamma_{e}$. Therefore, while the overall magnitude of the electronphonon coupling cannot be straightforwardly determined solely from the single-phonon RIXS signal, the momentum dependence of the coupling, as well as the dispersion, can be clearly read off directly from the RIXS intensity plots. In contrast, the magnitude of the coupling can be determine by the ratio of the phonon sidebands when multiple phonon inelastic events are considered [5,6].

Consideration of the interplay of electron-phonon coupling to the continuum, as well as the overall symmetry between the electron-phonon coupling and the $L$-edge RIXS process, can be seen clearly in Fig. 5, which shows the contribution to RIXS from the $B 1$ phonons (charge transfer between $\mathrm{O}_{x}$ and $\mathrm{O}_{y}$ in the $\mathrm{Cu}-\mathrm{O}$ unit cell). Both the

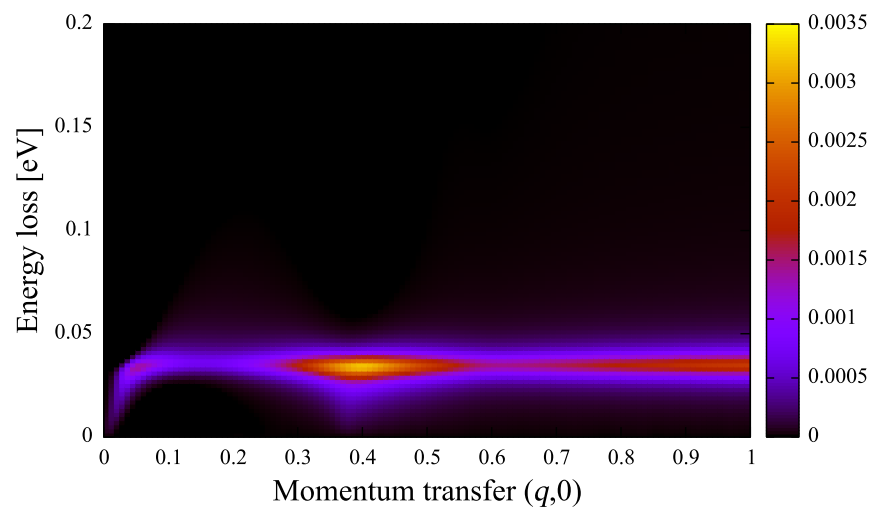

FIG. 5. $B 1$ phonon contribution to RIXS. Momentum transfer is given in units of $\pi / a$. overall dispersion and the intensity vary nonmonotonically across the BZ. We first consider symmetry effects. As already noted, this phonon branch at the zone center transforms as a lower representation of the $D^{4 h}$ point group; therefore, because of the locality of the RIXS process, the $B 1$ phonon branch does not contribute to the RIXS intensity at the zone center, and it grows for increasing momentum transfer. This result is in strong contrast to either resonant optical Raman scattering or infrared conductivity for which the intensities are strong for this phonon and show clear Fano interference with the particle-hole continuum [14].

Moreover, a clear interference effect is seen when the particle-hole continuum crosses the phonon line. This occurs weakly near the zone center and much more clearly around the weak nesting momentum $q \sim 0.4 \pi / a$. At both of these points, the phonon contribution to RIXS inherits contributions from the particle-hole continuum and shows a strong momentum-dependent Fano line shape, distorting along the lines of the soft continuum. The strength of this Fano effect is like that in Raman scattering, determined both by the strength of the electron-hole continuum at the frequency of the phonon and the magnitude of electronphonon coupling; however, in addition, the range of momentum transfers available through RIXS gives a clear qualitative way of understanding electron-lattice coupling near instabilities, allowing for the particle-hole continuum to interfere with the phonon in a narrow range of mode momenta.

This Fano effect is also indicated clearly in the $A 1$ phonon contribution to RIXS, shown in Fig. 6. Here, the symmetry of the $A 1$ modes (involving charge transfer between $\mathrm{Cu}-\mathrm{O}$ ) allows for finite coupling at the zone center, where the overall bare electron-phonon coupling is largest. However, a clear drop in intensity is observed where the electron-hole continuum crosses the phonon energy. This intensity would add to the intensity of the bare continuum shown in Fig. 3. The momentum-dependent

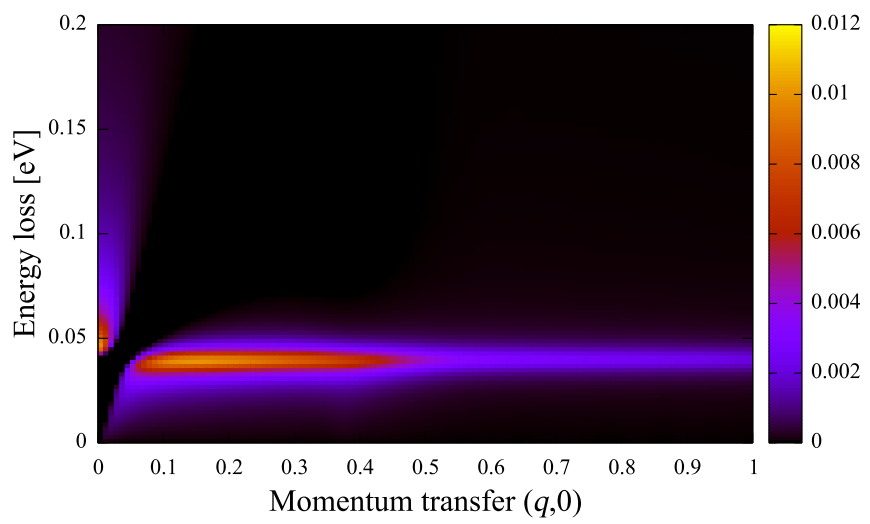

FIG. 6. A1 phonon contribution to RIXS. Momentum transfer is given in units of $\pi / a$. 


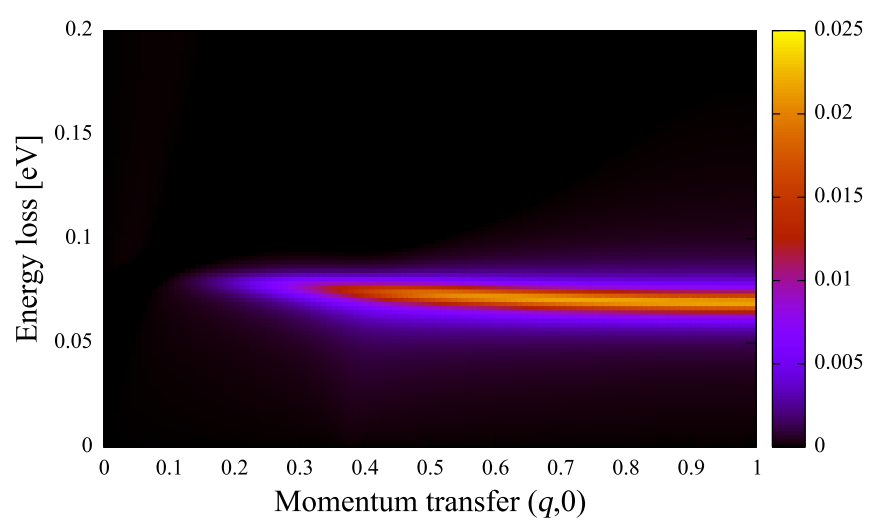

FIG. 7. Cu-O bond-stretching (breathing) phonon contribution to RIXS. Momentum transfer is given in units of $\pi / a$.

coupling falls off for large momentum transfers and gives vanishing intensity at the BZ boundary.

In Fig. 7, the $\mathrm{Cu}-\mathrm{O}$ bond-stretching phonons disperse downward away from the zone center, and because of the strong momentum dependence of the bare electron-phonon coupling, the intensity grows as $\sin ^{2}(q a / 2)$ so that it is largest at the BZ boundary. The coupling's momentum dependence minimizes the Fano effect near the BZ zone center yet allows for a weak coupling around the weak nesting feature near $q \sim 0.4 \pi / a$, where the intensity extends from the phonon line down to lower energy transfers.

In Fig. 8, the dispersionless apical oxygen contribution to RIXS is shown. The bare electron-phonon coupling is quite anisotropic in momentum space because of the charge transfer pathway between planar $\mathrm{Cu}$ and the apical oxygen, and it reaches its strongest coupling for the zone center. However, as in the case of $A 1$ phonons, the Fano effect is clearly observed at the intersection of the apical phonon with the main peak in the electron-hole continuum. The bare intensity would normally fall smoothly for larger momentum transfers, yet in contrast to the $A 1$ phonons and more like the $B 1$ phonons, the intensity remains large as the

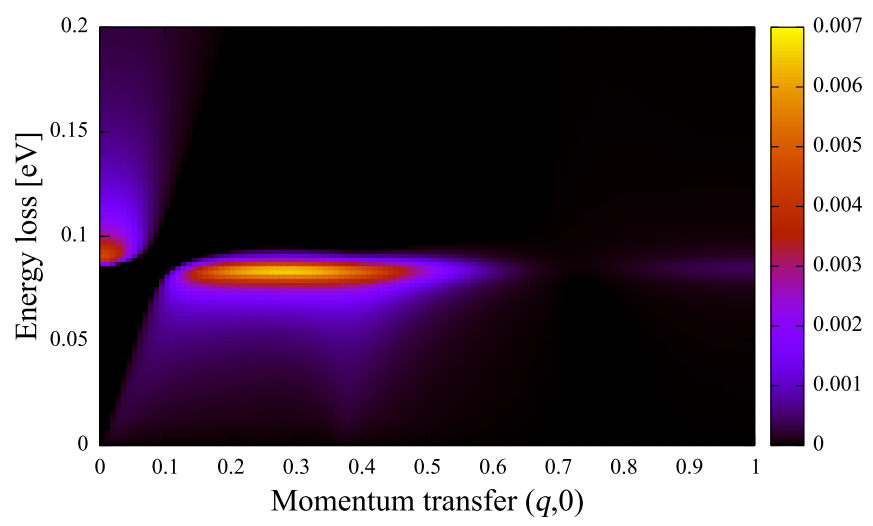

FIG. 8. Apical oxygen phonon contribution to RIXS. Momentum transfer is given in units of $\pi / a$.

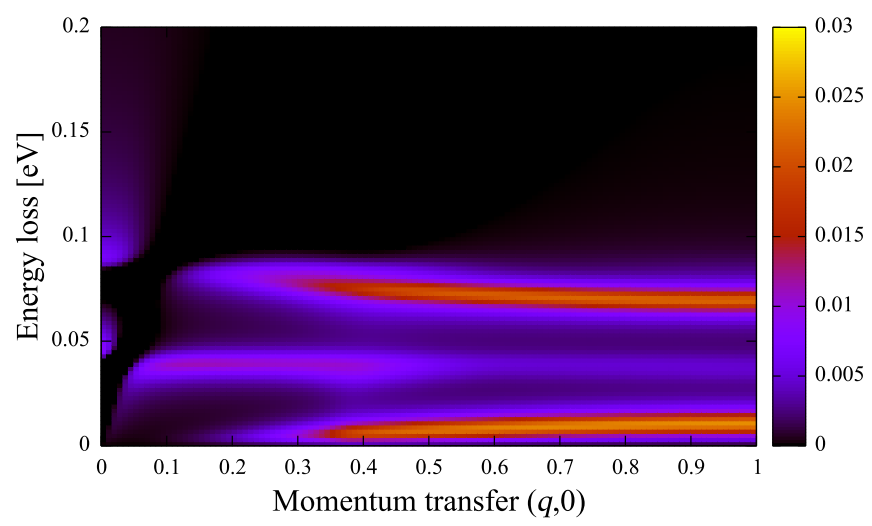

FIG. 9. Sum of all contributions from phonons to RIXS. Momentum transfer is given in units of $\pi / a$.

phonon line disperses across the weak nesting momentum before abruptly falling for larger momentum transfers.

As the electron-phonon coupling is only considered at lowest order, these contributions add to the overall RIXS signal, as shown in Fig. 9. One can clearly see that different phonon contributions are illuminated, depending upon the overall magnitude of the electron-phonon coupling as well as the intersection of the phonon line with the electron-hole continuum.

Finally, Fig. 10 plots the total RIXS intensity map from both the bare electron-hole continuum and the contributions from all phonon branches considered. The figure demonstrates the rich overall spectra having a strong momentum dependence. A detailed examination of the structure of the RIXS maps offers a unique perspective into the characteristics of lattice-electron coupling in a way which complements neutron and nonresonant $\mathrm{x}$-ray scattering, as well as Raman and infrared conductivity. Thus, RIXS at low energies offers a direct insight into couplings to lattice modes.

We add a remark concerning the effect of screening via the long-range Coulomb interaction, which has not been included in these considerations. It is well known that

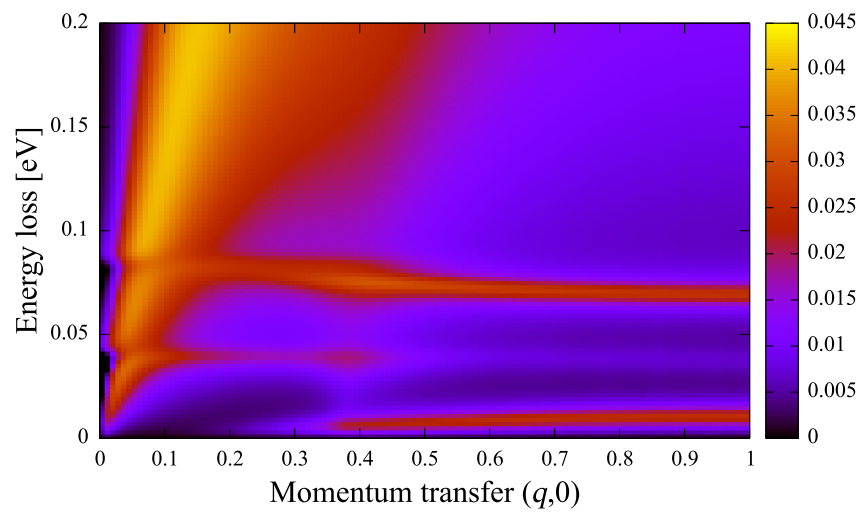

FIG. 10. Sum of all contributions. Momentum transfer is given in units of $\pi / a$. 
Coulomb screening mediates charge backflow that enforces particle-number conservation and can lead to strong modifications of the electron-phonon coupling for small momentum transfers, provided the symmetry of the coupling coincides with the full symmetry of the lattice $[15,28,29]$. In addition, in layer materials, a screening enhancement can occur when the phonon energy crosses the effective three-dimensional plasmon [11]. Both of these effects become prominent for small momentum transfers and do not affect the overall considerations given here for larger momentum transfers.

\section{SUMMARY AND CONNECTION TO EXPERIMENTS}

In this work, we have utilized a general formalism to investigate the way in which phonons can appear in a RIXS process. Our focus was specifically centered on the cuprates since a large majority of RIXS efforts to date have focused on the $\mathrm{Cu} L$-edge. With the advent of new RIXS instruments, RIXS is now in a strong position to investigate the phonons in cuprates and their coupling to the electronic degrees of freedom. Moreover, cuprates typically have large unit cells, which permit a panoply of phonon modes of many varieties that can couple differently to valence electrons. We have explicitly investigated a number of different types of phonon branches that couple differently to electrons. As the unit cells in the cuprates admit modes that are generic to any transitionmetal perovskite, our work is applicable to transition metal edge RIXS in a variety of compounds.

With the recent development of $\mathrm{x}$-ray sources and synchrotron facilities, two significant improvements have made RIXS a possible approach for directly addressing phonon excitations. First, at the ESRF, RIXS at the transition-metal $L$-edge has reached an energy resolution down to $35 \mathrm{meV}$, in the most difficult case $(\mathrm{Cu}-L)$ among $3 d$ elements, enabling RIXS to study not only the specific phonon modes on top of the $d d$ excitations or at the oxygen $K$-edge, but also the momentum-dependent one-phonon contributions from different phonon modes directly [38]. Second, although polarization discrimination has previously been limited to only the incoming photon, the recently constructed ESRF RIXS facility has made fully distinguishable polarization measurement for both incoming and outgoing photons possible [39]. Other end stations would also allow for the full photon polarization discrimination in the near future. Full polarization discrimination measurements can disentangle spin-flip, or single (para) magnon, excitations when the incoming and outgoing photon polarizations are perpendicular to one another (or cross-polarization) and non-spin-flip or electron-hole excitations when the incoming and outgoing photon polarizations are parallel to each other (or parallel polarization). If only polarization is utilized on the incoming photons, then a superposition of spin-flip and non-spin-flip excitations will be obtained. This can be partially mitigated by an appropriate choice of scattering configurations, but a full polarization dependence using both incident and scattered polarizations is needed to provide clearer discrimination of non-spin-flip phonon excitations or those that couple more directly with magnons.

To summarize, we have derived and calculated the electron-hole and one-phonon contributions to direct RIXS, specifically at the $\mathrm{Cu} L$-edge, based on a simple diagrammatic approach. We have examined an eight-band $\mathrm{Cu}-\mathrm{O}$ model with oxygen phonon modes, including $\mathrm{Cu}-\mathrm{O}$ planar longitudinal acoustic, $\mathrm{Cu}-\mathrm{O}$ bond-stretching, and apical $\mathrm{O}$ modes. This model reveals that the momentum dependence of the electron-phonon coupling can be extracted from the intensity of RIXS excitations in the low-energy regime, touting RIXS as a potential method to directly characterize the momentum-dependent electronphonon coupling. Our theoretical approach provides an intuitive picture of how different phonon modes contribute to RIXS and interact with charge densities, making RIXS a potential new technique for electron-phonon coupling measurement, complementary to ARPES, inelastic neutron scattering, and nonresonant inelastic x-ray scattering.

\section{ACKNOWLEDGMENTS}

This research was supported by the U.S. Department of Energy (DOE), Office of Basic Energy Sciences, Division of Materials Sciences and Engineering, under Contract No. DE-AC02-76SF00515, as well as by the SLAC National Accelerator Laboratory (SLAC), Stanford Institute for Materials and Energy Sciences. K. W. acknowledges support from the Polish National Science Center (NCN) under Project No. 2012/04/A/ST3/00331.

[1] E. Fradkin, S. A. Kivelson, and J. M. Tranquada, Theory of Intertwined Orders in High Temperature Superconductors, Rev. Mod. Phys. 87, 457 (2015).

[2] L. Ament, M. van Veenendaal, T. Devereaux, J. Hill, and J. van den Brink, Resonant Inelastic X-Ray Scattering Studies of Elementary Excitations, Rev. Mod. Phys. 83, 705 (2011).

[3] L. Braicovich, J. van den Brink, V. Bisogni, M. Moretti Sala, L. J. P. Ament, N. B. Brookes, G. M. De Luca, M. Salluzzo, T. Schmitt, V. N. Strocov, and G. Ghiringhelli, Magnetic Excitations and Phase Separation in the Underdoped $\mathrm{La}_{2-x} \mathrm{Sr}_{x} \mathrm{CuO}_{4}$ Superconductor Measured by Resonant Inelastic X-Ray Scattering, Phys. Rev. Lett. 104, 077002 (2010); M. Dean et al., Persistence of Magnetic Excitations in $\mathrm{La}_{2-x} \mathrm{Sr}_{x} \mathrm{CuO}_{4}$ from the Undoped Insulator to the Heavily Overdoped Non-superconducting Metal, Nat. Mater. 12, 1019 (2013); M. Le Tacon et al., Intense Paramagnon Excitations in a Large Family of High-Temperature Superconductors, Nat. Phys. 7, 725 (2011); K.-J. Zhou et al., Persistent high-energy spin excitations in iron-pnictide superconductors, Nat. Commun. 4, 1470 (2013). 
[4] V. N. Strocov et al., High-Resolution Soft X-Ray Beamline ADRESS at the Swiss Light Source for Resonant Inelastic $X$-Ray Scattering and Angle-Resolved Photoelectron Spectroscopies, J. Synchrotron Radiat. 17, 631 (2010).

[5] W. S. Lee et al., Role of Lattice Coupling in Establishing Electronic and Magnetic Properties in Quasi-OneDimensional Cuprates, Phys. Rev. Lett. 110, 265502 (2013).

[6] J. J. Lee et al., Charge-Orbital-Lattice Coupling Effects in the dd-Excitation Profile of One Dimensional Cuprates, Phys. Rev. B 89, 041104(R) (2014).

[7] G. Ghiringhelli, A. Piazzalunga, C. Dallera, G. Trezzi, L. Braicovich, T. Schmitt, V. N. Strocov, R. Betemps, L. Patthey, X. Wang, and M. Grioni, SAXES, a High Resolution Spectrometer for Resonant X-Ray Emission in the 400$1600 \mathrm{eV}$ Energy Range, Rev. Sci. Instrum. 77, 113108 (2006).

[8] N. Brookes, L. Braicovich, G. Ghiringhelli et al. (to be published).

[9] N. Bulut and D. J. Scalapino, $d_{x 2-y 2}$ Symmetry and the Pairing Mechanism, Phys. Rev. B 54, 14971 (1996).

[10] S. Y. Savrasov and O. K. Andersen, Linear-Response Calculation of the Electron-Phonon Coupling in Doped $\mathrm{CaCuO}_{2}$, Phys. Rev. Lett. 77, 4430 (1996).

[11] S. Johnston, F. Vernay, B. Moritz, Z.-X. Shen, N. Nagaosa, J. Zaanen, and T.P. Devereaux, Systematic Study of Electron-Phonon Coupling to Oxygen Modes Across the Cuprates, Phys. Rev. B 82, 064513 (2010).

[12] J. J. Lee, F. T. Schmitt, R. G. Moore, Y. T. Cui, W. Li, M. Yi, Z. K. Liu, M. Hashimoto, Y. Zhang, D. H. Lu, T.P. Devereaux, D.-H. Lee, and Z.-X. Shen, Interfacial Mode Coupling as the Origin of the Enhancement of $\mathrm{T}_{c}$ in FeSe Films on $\mathrm{SrTiO}_{3}$, Nature (London) 515, 245 (2014).

[13] M. D. Johannes and I. I. Mazin, Fermi Surface Nesting and the Origin of Charge Density Waves in Metals, Phys. Rev. B 77, 165135 (2008); H.-M. Eiter, M. Lavagnini, R. Hackl, E. A. Nowadnick, A. F. Kemper, T. P. Devereaux, J.-H. Chu, J. G. Analytis, I. R. Fisher, and L. Degiorgi, Alternative Route to Charge Density Wave Formation in Multiband Systems, Proc. Natl. Acad. Sci. U.S.A. 110, 64 (2013).

[14] M. Opel, R. Hackl, T. P. Devereaux, A. Virosztek, A. Zawadowski, A. Erb, E. Walker, H. Berger, and L. Forró, Physical Origin of the Buckling in $\mathrm{CuO}_{2}$ : Electron-Phonon Coupling and Raman Spectra, Phys. Rev. B 60, 9836 (1999).

[15] T. P. Devereaux and R. Hackl, Inelastic Light Scattering from Correlated Electrons, Rev. Mod. Phys. 79, 175 (2007); T. P. Devereaux and A.P. Kampf, Raman Scattering in Cuprate Superconductors, Int. J. Mod. Phys. B 11, 2093 (1997).

[16] T. P. Devereaux, A. Virosztek, and A. Zawadowski, Neutron Scattering and the $\mathrm{B}_{1 g}$ Phonon in the Cuprates, Phys. Rev. B 59, 14618 (1999); Charge-Transfer Fluctuation, d-Wave Superconductivity, and the $\mathrm{B}_{1 g}$ Raman Phonon in Cuprates, 51, 505 (1995).

[17] A. Damascelli, Z. Hussain, and Z.-X. Shen, Angle-Resolved Photoemission Studies of the Cuprate Superconductors, Rev. Mod. Phys. 75, 473 (2003); L. Pintschovius, ElectronPhonon Coupling Effects Explored by Inelastic Neutron Scattering, Phys. Status Solidi (b) 242, 30 (2005).
[18] T. Cuk, D. H. Lu, X. J. Zhou, Z.-X. Shen, T. P. Devereaux, and N. Nagaosa, A Review of Electron-Phonon Coupling Seen in the High-Tc Superconductors by Angle-Resolved Photoemission Studies (ARPES), Phys. Status Solidi (b) 242, 11 (2005).

[19] M. Maschek, S. Rosenkranz, R. Heid, A. H. Said, P. Giraldo-Gallo, I. R. Fisher, and F. Weber, Wave-VectorDependent Electron-Phonon Coupling and the ChargeDensity-Wave Transition in $\mathrm{TbTe}_{3}$, Phys. Rev. B 91, 235146 (2015).

[20] D. Reznik, L. Pintschovius, M. Ito, S. Iikubo, M. Sato, H. Goka, M. Fujita, K. Yamada, G. D. Gu, and J. M. Tranquada, Electron-Phonon Coupling Reflecting Dynamic Charge Inhomogeneity in Copper Oxide Superconductors, Nature (London) 440, 1170 (2006); S. R. Park, T. Fukuda, A. Hamann, D. Lamago, L. Pintschovius, M. Fujita, K. Yamada, and D. Reznik, Evidence for a Charge Collective Mode Associated with Superconductivity in Copper Oxides from Neutron and X-Ray Scattering Measurements of $\mathrm{La}_{2-x} \mathrm{Sr}_{x} \mathrm{CuO}_{4}$, Phys. Rev. B 89, 020506(R) (2014); M. Le Tacon, A. Bosak, S. M. Souliou, G. Dellea, T. Loew, R. Heid, K.-P. Bohnen, G. Ghiringhelli, M. Krisch, and B. Keimer, Inelastic X-Ray Scattering in $\mathrm{YBa}_{2} \mathrm{Cu}_{3} \mathrm{O}_{6.6}$ Reveals Giant Phonon Anomalies and Elastic Central Peak Due to Charge-Density-Wave Formation, Nat. Phys. 10, 52 (2014); C. J. Bonnoit, D. R. Gardner, R. Chisnell, A. H. Said, Y. Okada, T. Kondo, T. Takeuchi, H. Ikuta, D. E. Moncton, and Y. S. Lee, Probing Electronic Order via Coupling to Low Energy Phonons in Superconducting $\mathrm{Bi}_{2} \mathrm{Sr}_{2-x} \mathrm{La}_{x} \mathrm{CuO}_{6+\delta}$, arXiv:1202.4994.

[21] M. Braden, L. Pintschovius, T. Uefuji, and K. Yamada, Dispersion of the High-Energy Phonon Modes in $\mathrm{Nd}_{1.85} \mathrm{Ce}_{0.15} \mathrm{CuO}_{4}$, Phys. Rev. B 72, 184517 (2005); J. Graf, M. dAstuto, C. Jozwiak, D. R. Garcia, N. L. Saini, M. Krisch, K. Ikeuchi, A. Q. R. Baron, H. Eisaki, and A. Lanzara, Bond Stretching Phonon Softening and Kinks in the Angle-Resolved Photoemission Spectra of Optimally Doped $\mathrm{Bi}_{2} \mathrm{Sr}_{1.6} \mathrm{La}_{0.4} \mathrm{Cu}_{2} \mathrm{O}_{6+\delta}$ Superconductors, Phys. Rev. Lett. 100, 227002 (2008).

[22] S. Johnston, I. M. Vishik, W. S. Lee, F. Schmitt, S. Uchida, K. Fujita, S. Ishida, N. Nagaosa, Z.X. Shen, and T. P. Devereaux, Evidence for the Importance of Extended Coulomb Interactions and Forward Scattering in Cuprate Superconductors, Phys. Rev. Lett. 108, 166404 (2012).

[23] S. Moser, S. Fatale, P. Krüger, H. Berger, P. Bugnon, A. Magrez, H. Niwa, J. Miyawaki, Y. Harada, and Grioni, Electron-Phonon Coupling in the Bulk of Anatase $\mathrm{TiO}_{2}$ Measured by Resonant Inelastic X-Ray Spectroscopy, Phys. Rev. Lett. 115, 096404 (2015); Y. Y. Peng et al., Magnetic Excitations and Phonons Simultaneously Studied by Resonant Inelastic X-Ray Scattering in Optimally Doped $\mathrm{Bi}_{1.5} \mathrm{~Pb}_{0.55} \mathrm{Sr}_{1.6} \mathrm{La}_{0.4} \mathrm{CuO}_{6+\delta}$, Phys. Rev. B 92, 064517 (2015).

[24] L. J. P. Ament, M. van Veenendaal, and J. van den Brink, Determining the Electron-Phonon Coupling Strength from Resonant Inelastic X-ray Scattering at Transition Metal L-Edges, Euro. Phys. Lett. 95, 27008 (2011).

[25] Y. Wang, B. Moritz, C.-C. Chen, C. Jia, M. van Veenendaal, and T. P. Devereaux, Using Nonequilibrium Dynamics to 
Probe Competing Orders in a Mott-Peierls System, Phys. Rev. Lett. 116, 086401 (2016).

[26] A. S. Mishchenko, N. Nagaosa, K. M. Shen, Z.-X. Shen, X. J. Zhou, and T. P. Devereaux, Polaronic Metal in Lightly Doped High- $\mathrm{T}_{c}$ Cuprates, Europhys. Lett. 95, 57007 (2011).

[27] S. Johnston et al., Electron-Lattice Interactions Strongly Renormalize the Charge-Transfer Energy in the SpinChain Cuprate $\mathrm{Li}_{2} \mathrm{CuO}_{2}$, Nat. Commun. 7, 10563 (2016).

[28] T. P. Devereaux, D. Einzel, B. Stadlober, R. Hackl, D. H. Leach, and J. J. Neumeier, Electronic Raman Scattering in High $-\mathrm{T}_{c}$ Superconductors: A Probe of $\mathrm{d}_{x 2-y 2}$ Pairing, Phys. Rev. Lett. 72, 396 (1994); T. P. Devereaux, D. Einzel, B. Stadlober, and R. Hackl, Phys. Rev. Lett. 72, 3291 (1994).

[29] T. P. Devereaux, A. Virosztek, and A. Zawadowski, Multiband Electronic Raman Scattering in Bilayer Superconductors, Phys. Rev. B 54, 12523 (1996).

[30] A. M. Shvaika, O. Vorobyov, J. K. Freericks, and T. P. Devereaux, Resonant Enhancement of Inelastic Light Scattering in Strongly Correlated Materials, Phys. Rev. Lett. 93, 137402 (2004); Electronic Raman Scattering in Correlated Materials: A Treatment of Nonresonant, Mixed, and Resonant Scattering Using Dynamical Mean-Field Theory, Phys. Rev. B 71, 045120 (2005).

[31] P. Marra, K. Wohlfeld, and J. van den Brink, Unraveling Orbital Correlations with Magnetic Resonant Inelastic X-Ray Scattering, Phys. Rev. Lett. 109, 117401 (2012).
[32] C. Jia, K. Wohlfeld, Y. Wang, B. Moritz, and T. P. Devereaux, Using RIXS to Uncover Elementary Charge and Spin Excitations, Phys. Rev. X 6, 021020 (2016).

[33] G. Mahan, Many-Particle Physics, 3rd ed. (Springer, New York, 2000).

[34] F. M. F. de Groot, P. Kuiper, and G. A. Sawatzky, Local Spin-Flip Spectral Distribution Obtained by Resonant X-Ray Raman Scattering, Phys. Rev. B 57, 14584 (1998).

[35] M. van Veenendaal, Polarization Dependence of $L$ - and M-Edge Resonant Inelastic X-Ray Scattering in TransitionMetal Compounds, Phys. Rev. Lett. 96, 117404 (2006).

[36] Luuk J. P. Ament, G. Ghiringhelli, M. M. Sala, L. Braicovich, and J. van den Brink, Theoretical Demonstration of How the Dispersion of Magnetic Excitations in Cuprate Compounds Can Be Determined Using Resonant Inelastic X-Ray Scattering, Phys. Rev. Lett. 103, 117003 (2009).

[37] M. W. Haverkort, Theory of Resonant Inelastic X-Ray Scattering by Collective Magnetic Excitations, Phys. Rev. Lett. 105, 167404 (2010).

[38] See http://www.esrf.eu/UsersAndScience/Experiments/EMD/ ID32.

[39] M. Minola et al., Collective Nature of Spin Excitations in Superconducting Cuprates Probed by Resonant Inelastic X-Ray Scattering, Phys. Rev. Lett. 114, 217003 (2015); L. Braicovich et al., The Simultaneous Measurement of Energy and Linear Polarization of the Scattered Radiation in Resonant Inelastic Soft X-Ray Scattering, Rev. Sci. Instrum. 85, 115104 (2014). 